\title{
Enhanced transmission of microwave radiation in one-dimensional metallic gratings with subwavelength aperture
}

S. Sena Akarca-Biyikli, Irfan Bulu, and Ekmel Ozbay

Citation: Appl. Phys. Lett. 85, 1098 (2004); doi: 10.1063/1.1783013

View online: http://dx.doi.org/10.1063/1.1783013

View Table of Contents: http://aip.scitation.org/toc/apl/85/7

Published by the American Institute of Physics

\section{Articles you may be interested in}

Optical beam focusing by a single subwavelength metal slit surrounded by chirped dielectric surface gratings Applied Physics Letters 92, 013103 (2008); 10.1063/1.2828716

Gratingless enhanced microwave transmission through a subwavelength aperture in a thick metal plate Applied Physics Letters 81, 4661 (2002); 10.1063/1.1527704

Adaptable metasurface for dynamic anomalous reflection

Applied Physics Letters 110, 201904 (2017); 10.1063/1.4983782

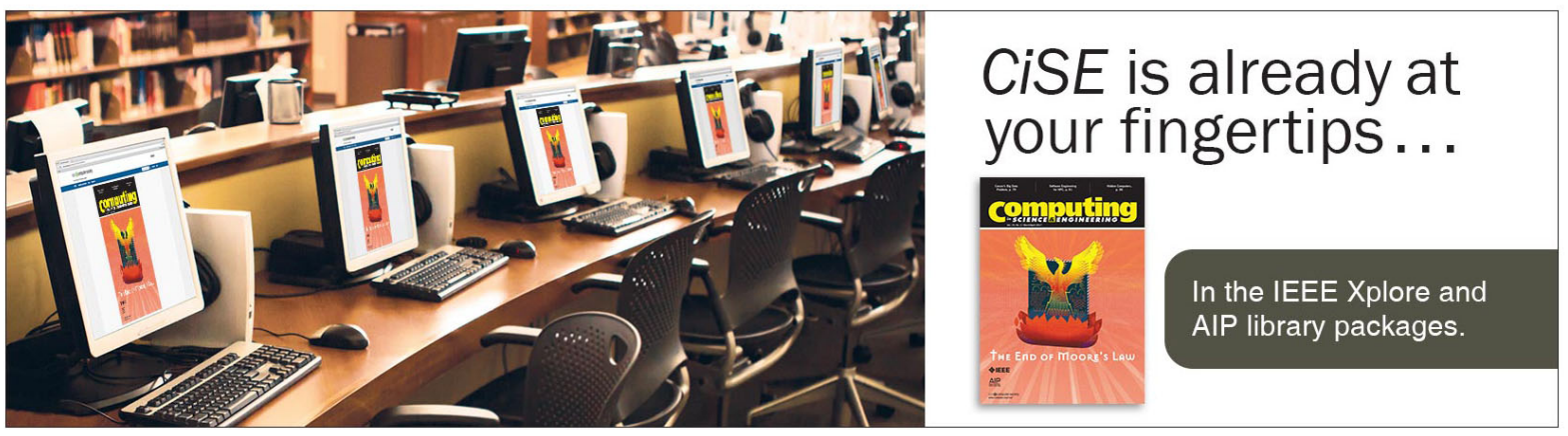




\title{
Enhanced transmission of microwave radiation in one-dimensional metallic gratings with subwavelength aperture
}

\author{
S. Sena Akarca-Biyikli, ${ }^{a)}$ Irfan Bulu, and Ekmel Ozbay \\ Department of Physics, Bilkent University, Bilkent, Ankara 06800, Turkey
}

(Received 5 April 2004; accepted 17 June 2004)

\begin{abstract}
We report a theoretical and experimental demonstration of enhanced microwave transmission through subwavelength apertures in metallic structures with double-sided gratings. Three different types of aluminum gratings (sinusoidal, symmetric rectangular, and asymmetric rectangular shaped) are designed and analyzed. Our samples have a periodicity of $16 \mathrm{~mm}$, and a slit width of $2 \mathrm{~mm}$. Transmission measurements are taken in the $10-37.5 \mathrm{GHz}$ frequency spectrum, which corresponds to $8-30 \mathrm{~mm}$ wavelength region. All three structures display significantly enhanced transmission around surface plasmon resonance frequencies. The experimental results agree well with finite-difference-time-domain based theoretical simulations. Asymmetric rectangular grating structure exhibits the best results with $\sim 50 \%$ transmission at $20.7 \mathrm{~mm}$, enhancement factor of $\sim 25$, and $\pm 4^{\circ}$ angular divergence. (C) 2004 American Institute of Physics. [DOI: 10.1063/1.1783013]
\end{abstract}

Extraordinary transmission through subwavelength apertures has inspired great interest since the work of Ebbesen $e t$ $a l .{ }^{1}$ They reported orders of magnitude enhancement in optical wavelengths, through subwavelength hole arrays due to the coupling of light with surface plasmons (SPs). Surface plasmons are nonradiative electromagnetic waves on the metallic surfaces and can couple with photons by surface corrugations. $^{2}$ Recently enhanced transmission ${ }^{3,4}$ and beaming of light ${ }^{5}$ through a single aperture by periodic surface corrugations around the aperture were shown experimentally and studied theoretically. ${ }^{6,7}$ Also enhanced transmission through subwavelength hole arrays was shown at $\mathrm{THz}$ radiation. $^{8}$

Similar studies were carried out at microwave frequencies by Hibbins et al. recently. Using grating structures, they have demonstrated the coupling of microwave radiation to SPs by reflection measurements. ${ }^{9,10}$ They have also presented microwave transmission enhancement through a subwavelength slit without grating. ${ }^{11}$ Very recently, Lockyear et al. have reported enhanced transmission at microwave frequencies through circular subwavelength aperture surrounded by periodic concentric grooves. ${ }^{12}$ Enhanced microwave transmission in one-dimensional grating structures with subwavelength slit apertures was not reported previously. In this work, we experimentally and theoretically demonstrate transmission enhancement of microwave radiation through a subwavelength slit by adding grating on both surfaces of the metallic structure.

Schematic description of the first sample (sample A) is drawn in Fig. 1(a). The sample consists of a slit and periodic sinusoidal grating on both surfaces around the slit. Aluminum is used as metal. Slit thickness $(w)$ and slit width $(a)$ are 8 and $2 \mathrm{~mm}$, respectively. Period of grating $\left(\lambda_{g}\right)$ is $16 \mathrm{~mm}$ and groove depth $(h)$ is $4 \mathrm{~mm}$. The experimental setup consists of an HP 8510C network analyzer and two horn antennas to measure the transmission amplitude. Horn antennas are in TM mode so that electric field is perpendicular to the slit and gratings. Radiation is normally incident upon the sample. Receiver antenna is $30 \mathrm{~cm}$ away from the sample's

\footnotetext{
${ }^{a)}$ Electronic mail: sbiyikli@fen.bilkent.edu.tr
}

back face and it is connected to a rotating arm to measure angle dependence of the far-field radiation. Theoretical calculations are done using finite-difference-time domain method based simulations. We model our structure by using Drude dispersion model, i.e.,

$$
\varepsilon(\omega)=1-\frac{\omega_{p}^{2}}{\omega\left(\omega-i \omega_{\tau}\right)},
$$

where $\omega_{p}=3570 \mathrm{THz}$ is the plasma frequency and $\omega_{\tau}$ $=19.4 \mathrm{THz}$ is the absorption coefficient for aluminum. ${ }^{13}$

Figure 1(b) shows the experimental and simulation results of transmission spectra of reference sample (metal plate with a $2 \mathrm{~mm}$ width slit) and sample A. The experimental results are in good agreement with our theoretical calculations. For the single slit case, two peaks are calculated at 11.3 and $24.3 \mathrm{GHz}$. These peaks correspond to the slit waveguide resonances. ${ }^{7}$ Measured peaks are slightly blueshifted; at 12.6 and $26.5 \mathrm{GHz}$. Transmission peaks corresponding to
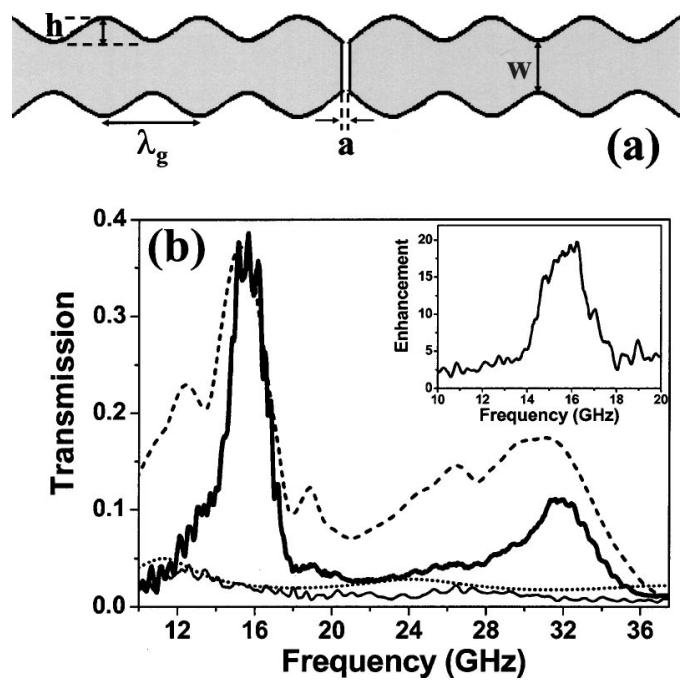

FIG. 1. Schematic of sample A with $\lambda_{g}=16 \mathrm{~mm}, a=2 \mathrm{~mm}, w=8 \mathrm{~mm}$, and $h=4 \mathrm{~mm}$. (b) Measured transmission spectrum of sample A (thick solid line), reference sample (thin solid line), simulated transmission spectrum of sample A (dashed line), reference sample (dotted line). Inset shows the enhancement factor obtained with sample A. 
the slit modes are very low when compared to the SP resonant peaks in grating samples. For sample A, two transmission peaks are measured at frequencies 15.6 and $31.6 \mathrm{GHz}$, with amplitudes of 0.41 and 0.11 , respectively. Theoretical simulations predict slightly redshifted similar transmission peaks: $37 \%$ at $15.2 \mathrm{GHz}$ and $17 \%$ at $31.1 \mathrm{GHz}$. Coupling of incident radiation to the surface plasmon waves on the grating structure is given by

$$
\mathbf{k}_{\mathrm{SP}}=n_{d} \mathbf{k}_{0} \sin \theta \pm N \mathbf{k}_{g} .
$$

In this equation $\mathbf{k}_{\mathrm{SP}}$ is surface plasmon wave vector, $n_{d}$ is refractive index of dielectric (here $n_{d}=1$ for air), $\mathbf{k}_{0}$ is incident wave vector, $\theta$ is the incidence angle, $N$ is an integer, and $\mathbf{k}_{g}=2 \pi / \lambda_{g}$ is grating wave vector where $\lambda_{g}$ is grating period. SP wave vector is calculated by

$$
k_{\mathrm{SP}}=k_{0} \sqrt{\frac{\varepsilon_{m} \varepsilon_{d}}{\varepsilon_{m}+\varepsilon_{d}}},
$$

where $\varepsilon_{m}$ and $\varepsilon_{d}$ denote the permittivity of metallic and dielectric media, respectively. For microwave frequencies permittivity of metals are really high $\left(\sim 10^{6}\right)$, compared to the permittivity of air; so $k_{\mathrm{SP}}$ is close to $k_{0}$ in this range. Since the radiation is normally incident to the grating, coupled wave vector should be the multiples of $\mathbf{k}_{g}$. In our sample $\lambda_{g}$ is $16 \mathrm{~mm}$ and coupled frequencies are expected to be about $c / \lambda_{g}=18.75 \mathrm{GHz}$ and integer multiples. However, in our experiments and simulations, coupled frequencies shifted to lower frequencies $(15.6,31.6 \mathrm{GHz})$ due to the retardation by metal. ${ }^{4}$ To investigate the effect of grating period, we tried $32 \mathrm{~mm}$ grating period. Since the period is doubled, the first resonant frequency should be half of the resonant frequency of the sample with the $16 \mathrm{~mm}$ period. In transmission spectrum, peaks at $7.9 \mathrm{GHz}(N=1), \quad 15.6 \mathrm{GHz}(N=2)$, and $23.8 \mathrm{GHz}(N=3)$ are observed as expected. A detailed investigation of the impact of grating period will be presented elsewhere.

Enhancement is calculated according to the reference sample. A maximum enhancement factor of $\sim 20$ is achieved around the first resonance frequency, which is shown in the inset of Fig. 1(b).

In optical wavelengths Lezec et al. showed experimental results for transmission through a single aperture and a single slit surrounded by surface corrugations. ${ }^{5}$ That corrugation was a symmetric rectangular grating. We try this kind of grating (sample B) instead of sinusoidal grating in the microwave regime. The period of the grating, grating depth, slit width, and slit thickness are the same as our first sample. Groove widths (b) are $8 \mathrm{~mm}$, half of the period [Fig. 2(a)]. Transmission spectrum of sample B is seen in Fig. 2(b). There are two resonance peaks - the first one is at $14.6 \mathrm{GHz}$ with transmission efficiency of $45 \%$. The dashed line shows the results of simulation that is in good agreement with the experimental values. When compared with sample A, transmission efficiency of sample B is higher and redshifted about $1 \mathrm{GHz}$. A broader transmission peak is measured with this sample. Enhancement is above 20, which is similar to sample A.

In a recent theoretical paper which explains the experimental results of Ref. 5, different grating structure with the same period was used. In that structure, groove widths are the same as the slit width. We also try this asymmetric rectangular structure in the microwave regime (sample $\mathrm{C}$ ). In
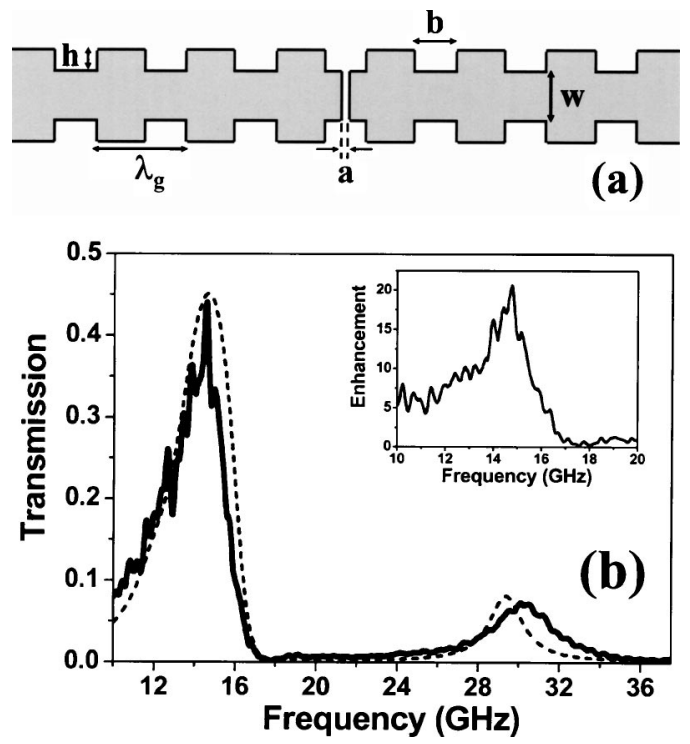

FIG. 2. (a) Schematic of sample B with $\lambda_{g}=16 \mathrm{~mm}, a=2 \mathrm{~mm}, b=2 \mathrm{~mm}$, $w=8 \mathrm{~mm}$, and $h=4 \mathrm{~mm}$. (b) Measured and simulated transmission spectrum of sample B (solid line and dashed line, respectively). Inset shows the measured enhancement factor for sample B.

this sample, again the period of grating, groove depths, and slit width are the same. Groove widths are equal to the slit width, $2 \mathrm{~mm}$ [Fig. 3(a)]. The results of this structure are more promising-that the transmission peak is higher and narrower [Fig. 3(b)]. Resonance frequency is at $14.5 \mathrm{GHz}$, which is very close to the resonance frequency of sample B. Transmission efficiency is $\sim 50 \%$, which is larger than the theoretically predicted peak transmission efficiency. The enhancement factor is higher, about 25 , and confined to the resonant frequency. When we compare with other structures, enhancement figures of samples A and B are broader so that enhancement occurs within a broader frequency range. However with asymmetric square grating, enhancement is confined to a certain frequency determined by the period. Hence this type of asymmetric grating is more useful for applications where wavelength selectivity is important.
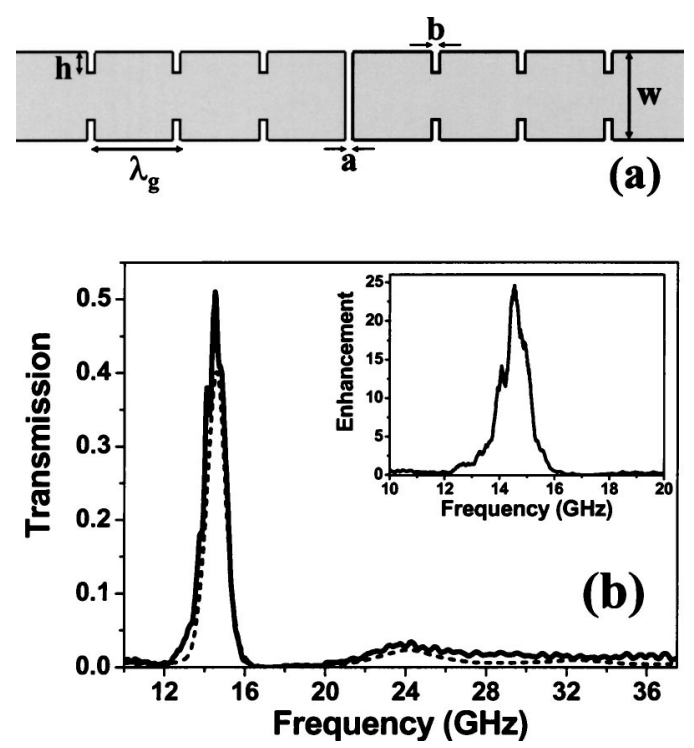

FIG. 3. (a) Schematic of sample $\mathrm{C}$ with $\lambda_{g}=16 \mathrm{~mm}, a=2 \mathrm{~mm}, b=2 \mathrm{~mm}$, $w=16 \mathrm{~mm}$, and $h=4 \mathrm{~mm}$. (b) Measured and simulated transmission spectrum of sample $\mathrm{C}$ (solid line and dashed line, respectively). Enhancement factor for sample $\mathrm{C}$ is shown in the inset. 


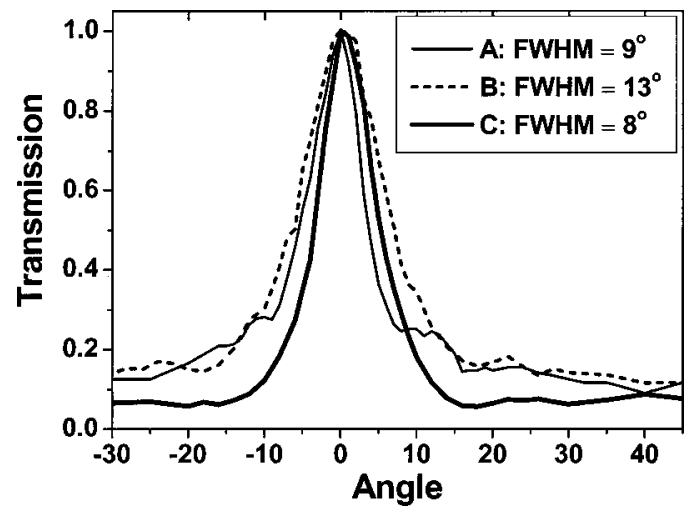

FIG. 4. Normalized transmission as a function of reception angle. The measurements were taken at the resonance frequency of each sample.

In order to prevent diffraction, in our experiments, we have used structures that have gratings on both surfaces. ${ }^{4}$ To compare the beaming effect of each grating, receiver antenna is attached to a rotating arm and transmission data at resonant frequencies are recorded as a function of angle by one degree increments. Figure 4 illustrates the normalized transmission intensity as a function of the reception angle. The measured transmission sharply decreases with increased angle. Sample $\mathrm{C}$ exhibits the highest directivity with a full width at half maximum (FWHM) of $8^{\circ}$. Samples A and B displayed FWHM values of $9^{\circ}$ and $13^{\circ}$, respectively.

In conclusion, experimental and numerical results of microwave transmission through a subwavelength slit $(\sim \lambda / 10)$ surrounded by metallic gratings on both surfaces have been presented. Different grating structures (sinusoidal, symmetric rectangular, asymmetric rectangular) have been designed, simulated, and measured. All three structures dis- play enhanced transmission at SP resonance frequencies. Our experimental results agree well with theoretical simulations. The highest transmission $(\sim 50 \%)$, enhancement $(\sim 25)$, and directivity (FWHM $\sim 8^{\circ}$ ) is achieved with asymmetric rectangular grating structure.

This work was supported by European Union under the projects EU-DALHM, EU NOE-METAMORPHOSE and by Turkish Department of Defense Grant No. KOBRA-002. One of the authors (E.O.) acknowledges partial support from Turkish Academy of Sciences.

${ }^{1}$ T. W. Ebbesen, H. J. Lezec, H. F. Ghaemi, T. Thio, and P. A. Wolf, Nature (London) 391, 667 (1998).

${ }^{2}$ H. Raether, Surface Plasmons (Springer, Berlin, 1988).

${ }^{3}$ D. E. Grupp, H. J. Lezec, T. Thio, and T. W. Ebbesen, Adv. Mater. (Weinheim, Ger.) 11, 860 (1999).

${ }^{4}$ T. Thio, K. M. Pellerin, R. A. Linke, H. J. Lezec, and T. W. Ebbesen, Opt. Lett. 26, 1972 (2001).

${ }^{5}$ H. J. Lezec, A. Degiron, B. Devaux, R. A. Linke, L. Martin-Moreno, F. J. Garcia-Vidal, and T. W. Ebbesen, Science 297, 820 (2002).

${ }^{6}$ L. Martin-Moreno, F. J. Garcia-Vidal, H. J. Lezec, A. Degiron, and T. W. Ebbesen, Phys. Rev. Lett. 90, 167401 (2003).

${ }^{7}$ F. J. Garcia-Vidal, H. J. Lezec, T. W. Ebbesen, and L. Martin-Moreno, Phys. Rev. Lett. 90, 213901 (2003)

${ }^{8}$ J. G. Rivas, C. Schotsch, P. H. Bolivar, and H. Kurz, Phys. Rev. B 68, 201306 (2003)

${ }^{9}$ A. P. Hibbins, J. R. Sambles, and C. R. Lawrence, J. Appl. Phys. 86, 1791 (1999).

${ }^{10}$ A. P. Hibbins, J. R. Sambles, and C. R. Lawrence, J. Appl. Phys. 87, 2677 (2000).

${ }^{11}$ A. P. Hibbins, C. R. Sambles, and C. R. Lawrence, Appl. Phys. Lett. 81, 4661 (2002).

${ }^{12}$ M. J. Lockyear, A. P. Hibbins, J. R. Sambles, and C. R. Lawrence, Appl. Phys. Lett. 84, 2040 (2004).

${ }^{13}$ M. M. Sigalas, C. T. Chan, K. M. Ho, and C. M. Soukoulis, Phys. Rev. B 52, 11744 (1995). 